\title{
Photonic studies on polymer-coated sapphire-spheres: A model system for biological ligands
}

\author{
M.S. Murib ${ }^{a}$, W.S. Yeap ${ }^{a}$, D. Martens ${ }^{b}$, X. Liu ${ }^{c}$, P. Bienstman ${ }^{\text {b }}$, M. Fahlman ${ }^{c}$, \\ M.J. Schöning ${ }^{\mathrm{d}}$, L. Michiels ${ }^{\mathrm{e}}, \mathrm{K}$. Haenen ${ }^{\mathrm{a}, \mathrm{f}}$, A. Serpengüzel ${ }^{\mathrm{g}}$, P. Wagner ${ }^{\mathrm{a}, \mathrm{f}, *}$ \\ a Hasselt University, Institute for Materials Research IMO, Wetenschapspark 1, B-3590 Diepenbeek, Belgium \\ b Photonics Research Group, Department of Information Technology, Ghent University - INTEC, Sint-Pietersnieuwstraat 41, B-9000 Gent, Belgium \\ ${ }^{\mathrm{c}}$ Linköping University, Department of Physics, Chemistry and Biology, S-581 83 Linköping, Sweden \\ d Aachen University of Applied Sciences, Institute of Nano-and Biotechnologies, Heinrich-Mußmann-Straße 1, D-52428 Jülich, Germany \\ ${ }^{\mathrm{e}}$ Hasselt University, Biomedical Research Institute BIOMED, Agoralaan, B-3590 Diepenbeek, Belgium \\ ${ }^{\mathrm{f}}$ IMEC vzw, IMOMEC, Wetenschapspark 1, B-3590 Diepenbeek, Belgium \\ ${ }^{g}$ Koç University, Microphotonics Research Laboratory, Department of Physics, Rumelifeneri Yolu, Sarıer, Istanbul 34450, Turkey
}

\section{Introduction}

Development of biological, chemical and biochemical sensors is one of the current needs of the society. Label-free detection of DNA was studied by elastic measurements at the level of a single DNA molecule[1] via force-induced denaturation [2], electrochemistry $[3,4]$, field-effect sensors [5,6], and by monitoring the denaturation dynamics in real-time using impedance spectroscopy [7] and the heat transfer method [8]. Label-free detection of protein-protein interaction was shown recently using nanowires [9], nanoparticle probes [10], biochips [11], and mechanical cantilevers [12]. Optical measurement techniques can provide high sensitivity, compactness, fast response and real-time measurements [13]. They are non-destructive to the sample, and the transduction processes generally take place on a surface and can be tailored to sense almost any kind of (bio)chemical molecules [14].

Optical sensing can be performed using plasmonic biosensors [15], ring resonators [16], confocal microscopy [7], prism couplers [17], and spherical cavities $[18,19]$. Optical microcavities such as spherical resonators, where optical rays are confined by total internal reflection, are promising optical label-free detection setups and play a prominent role in modern optics [20].

Recently, a silicon-on-insulator (SOI) microring resonator for sensitive label-free biosensing was fabricated [21]. Hereby, microring surface functionalization with biotin and a detection limit of $0.37 \mathrm{fg}$ avidin mass (3260 molecules) was achieved [22,23]. In an optical microcavity, the target molecules can be sampled hundreds of times due to the recirculation of light within the microcavity by total internal reflection (TIR) [20]. The target molecule induces a change in the optical microcavity properties such as its size. As a result, a change in the whispering gallery mode (WGM) resonant wavelength is encountered [24]. In order to preserve the high quality of the transducer and the interaction with the sensing layer and

* Corresponding author at: Catholic University Leuven, Department of Physics and Astronomy, Soft Matter and Biophysics, Celestijnenlaan 200 D, B-3001 Leuven, Belgium.

E-mail addresses: Patrick.Wagner@uhasselt.be,Patrick.Wagner@fys.kuleuven.be (P. Wagner) 
the WGMs [14,24-26] a good control of surface functionalization of the transducer surface is a crucial step for producing reliable biosensors for the binding of the biological recognition element to it. The circumnavigating light within the microcavity will evanesce to the external medium and extends about $200 \mathrm{~nm}$ [24]. Hence, the functional layer has to be very thin, i.e. $\approx 10$ to $\leq 200 \mathrm{~nm}$, and homogeneous.

So far, silicon-on-insulator ring resonators [23] and glass microspheres [18] have been used as microcavity-based biosensors. However, silicon and glass are known to degrade easily in aqueous media, especially at longer time scale and in non-neutral $\mathrm{pH}$ circumstances $[27,28]$. Hence, the usibilty of these two materials as biosensors is somehow limited. To overcome the drawback of limited chemical stabiltiy, recently, diamond ring resonators [29] and nearly spherical diamond resonators were developed [30]. However, their fabrication is expensive and diamond surface modification with proteins or DNA still requires a good control of the functionalization steps $[27,31]$.

In this article, an experimental study of an optical biosensor concept based on elastic light scattering from sapphire microspheres and the corresponding shift of WGMs, after an add-on polydopamine (PDA) layer to the sphere, is performed. Mussel-inspired polydopamine coating have been intensely studied in recent years [32-34]. Surface functionalization using this biopolymer is especially robust. By simply dipping any substrate into an aqueous solution of dopamine, a surface-adherent polydopamine thin film is first formed within hours through oxidative self-polymerization and subsequentially bounded to the sapphire surface via hydrogen bonds [32,35]. The thickness of the formed polymer can be tuned by varying the concentration of the dopamine used or by adapting the immersion time of the substrates. Using atomic force micrsocopy (AFM), Lee et al. found that the thickness of the polymers formed

(a)
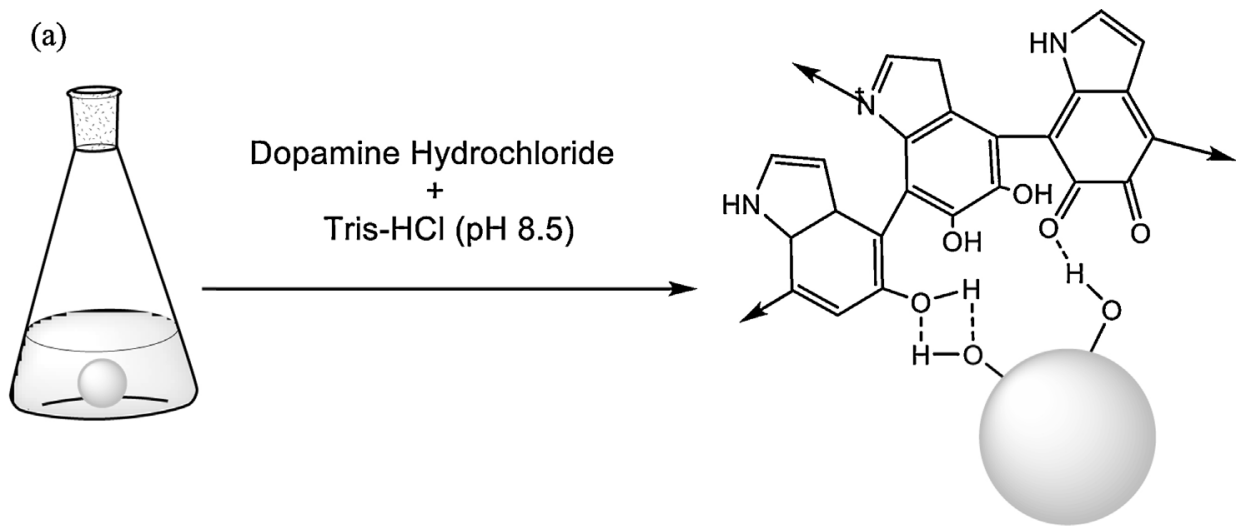

hydrogen bond

covalent bond

(b)

\section{Dopamine}<smiles>NCCc1ccc(O)c(O)c1</smiles>

Dopaminechrome<smiles>CC1=C2CCN=C2C=C(O)C1=O</smiles>

$\left(2 \mathrm{H}^{+}+2 \mathrm{e}^{-}\right)$ 5,6-dihydroxyindole

Polydopamine<smiles>NCCC1=CC(=O)C(=O)C=C1</smiles>

Dopaminequinone

Fig. 1. (a) Sapphire surface functionalization with PDA. (b) Polymerization mechanisms of dopamine adapted from Lee et al. 
on gold surfaces increases linearly with time at the beginning of the reaction and saturates after $20 \mathrm{~h}$ [32]. Polymers with similar thickness were also obtained by Pop-Georgievski et al. on hydrogen and oxygenated diamond surfaces measured by spectroscopic ellipsometry [35]. Various post-modification reactions are possible to create a variety of ad-layers on this polymer coating to fulfill various purposes [34,36-39]. WGMs have been used in polymeric microresonators [40]. Surface functionalization of this biopolymer on sapphire microspheres is an alternative to polymeric resonators and can be used as a platform for future bio-optical biosensing applications. In this article we therefore address sapphire, not only the second hardest material next to diamond but it also shows outstanding chemical inertness, wear resistance, and biocompatibility [41]. Hence, it is widely used for implants such as hip [41], dental [42], and endosseous implants [43]. Moreover, its wide optical transmission band from the ultraviolet (UV) to the near-infrared (near-IR) [44] suggests application potential in optical biosensors.

\section{Experimental method}

\subsection{Surface functionalization}

2-Amino-2-(hydroxymethyl)-1,3-propanediol hydrochloride (TRIS-HCl) (reagent grade, minimum 99\%) and dopamine hydrochloride (98.5\%) were purchased from Sigma and used as received. The formation of a polydopamine film was carried out by immersing the sapphire microsphere (Edmund Optics) into a solution of dopamine hydrochloride $(2 \mathrm{mg} / \mathrm{mL})$ in $10 \mathrm{mM}$ Tris- $\mathrm{HCl}$ buffer that was adjusted to $\mathrm{pH} 8.5$ by adding $0.5 \mathrm{M} \mathrm{NaOH}$. Immersion times were varied $(2 \mathrm{~h}, 5 \mathrm{~h}, 10 \mathrm{~h}$ and $20 \mathrm{~h})$ to create polydopamine films with different thicknesses [32]. To prevent the formation of microparticles in the dopamine solution, the solution together with the sapphire microsphere was stirred with a shaker during the reaction. After the reaction, the coated sapphire microsphere was thoroughly rinsed with deionized water and dried under a stream of nitrogen (Fig. 1a).

Fig. 1b presents a brief illustration of the polymerization mechanisms of dopamine adapted from Lee et al. [32]. Briefly, under an oxidative condition, e.g., alkaline buffer, dihydroxyl group protons in dopamine are deprotonated, becoming dopaminequinone. Dopamine-quinone is then rearranged via intramolecular cyclization to leukodopaminechrome. Further oxidation and rearrangement leads to 5,6-dihydroxyindole, which oxidation causes intermolecular cross-linking to yield a polymer that is structurally similar to the biopigment melanin [32].

\subsection{X-ray photoelectron spectroscopy (XPS)}

For reference purposes, X-ray photoelectron spectroscopy (XPS) measurements were performed on a single crystalline sapphire chip instead of sapphire sphere in order to confirm the presence of the PDA layer after polymerization. Given the respective dimensions of the molecules used in the PDA layer synthesis and the sapphire microsphere, the microsphere surface will appear "flat" to the molecules binding to the surface. The surface roughness of the sapphire microsphere and the sapphire chip are on the same order of magnitude, therefore the sapphire-chip XPS data can be applied to the sapphire microsphere. Moreover, sapphire chips have a well defined take-off angle. A sapphire chip $(1 \mathrm{~cm} \times 1 \mathrm{~cm})$ with crystalline orientation $[0,1,-1,2]$ as measured using X-ray diffraction (XRD) was used for the XPS measurements. It was functionalized with PDA ( $5 \mathrm{~h}$ immersion) just as described in Section 3. Section 2.1. Photoemission experiments were carried out using a Scienta ESCA 200 spectrometer in ultrahigh vacuum with a base pressure of $1 \times 10^{-10}$ mbar (Linköping University, Sweden). The

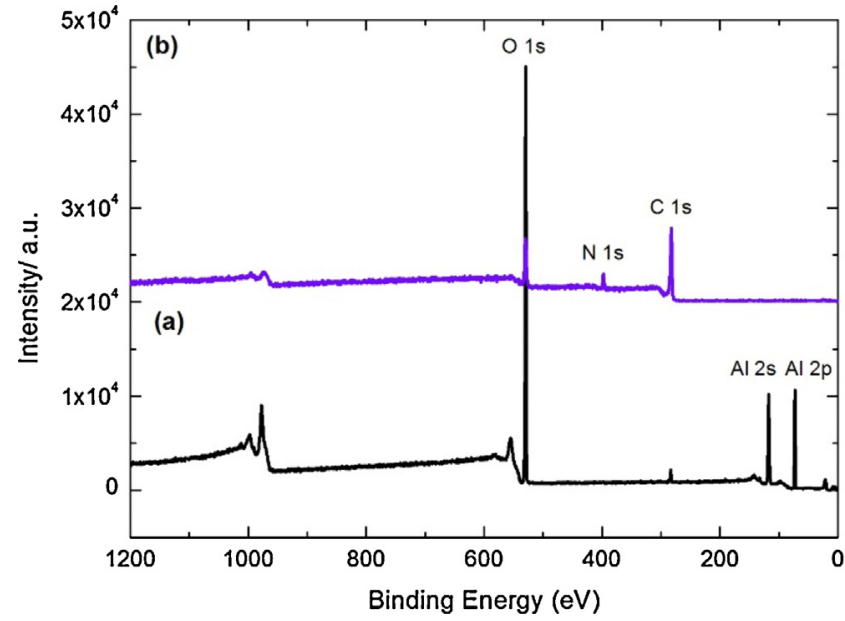

Fig. 2. XPS spectra of a sapphire chip before (a) and after (b) $5 \mathrm{~h}$ functionalization with PDA layer. The curves are displaced vertically for better clarity.

measurement chamber was equipped with a monochromatic $\mathrm{Al} \mathrm{K}$ X-ray source $(1486.6 \mathrm{eV})$. The XPS experimental conditions were set so that the full-width-at-half-maximum of the clean $\mathrm{Au} 4 \mathrm{f}_{7 / 2}$ line was $0.65 \mathrm{eV}$. All spectra were recorded under a photoelectron take-off angle of $0^{\circ}$, i.e. normal emission.

Spectrum a in Fig. 2 shows the XPS specrum of a blank sapphire chip. The blank sapphire shows three major peaks at $118 \mathrm{eV}$, $73 \mathrm{eV}$, and $532 \mathrm{eV}$, which corresponds to $\mathrm{Al} 2 \mathrm{~s}, \mathrm{Al} 2 \mathrm{p}$, and $\mathrm{O} 1 \mathrm{~s}$ peaks, respectively. A negligible $\mathrm{C} 1 \mathrm{~s}$ peak appears at $284 \mathrm{eV}$, which might be attributed to contamination. Simple immersion of substrates in a dilute aqueous solution of dopamine, buffered to a slightly basic $\mathrm{pH}$ ( $2 \mathrm{mg}$ of dopamine per milliliter of $10 \mathrm{mM}$ Tris- $\mathrm{HCl}$ buffer, $\mathrm{pH}$ 8.5), resulted in spontaneous deposition of a thin adherent polymer film. X-ray photoelectron spectroscopy (XPS) analysis of the sapphire chip coated during $5 \mathrm{~h}$ (Fig. 2 spectrum b) shows two additional peaks at $400 \mathrm{eV}$ and $284 \mathrm{eV}$ which are related to $\mathrm{N} 1 \mathrm{~s}$ and $\mathrm{C} 1 \mathrm{~s}$, respectively. The presence of these peakes can be attributed to the nitrogen $(\mathrm{N})$ and carbon $(\mathrm{C})$ atoms in the PDA layer. Moreover, XPS analysis also revealed the absence of signals specific to the sapphire chip ( $\mathrm{Al} 2 \mathrm{p}$ and $\mathrm{Al} 2 \mathrm{~s}$ peaks), indicating the formation of a polymer coating is greater than $10 \mathrm{~nm}$ in thickness [32]. We will show in Section 4 on paragraph 4 that $5 \mathrm{~h}$ of polymerization corresponds to a PDA layer of $25 \mathrm{~nm}$ thickness.

\subsection{Detection setup}

Fig. 3(a) shows the detection setup used in this experiment. The diode laser was tuned from $1510 \mathrm{~nm}$ to $1512 \mathrm{~nm}$ with a step size of $5 \mathrm{pm}$. The laser light was coupled to the sapphire sphere through a side polished single mode optical fiber (SMOF). When the resonances wavelength of the laser matches the resonances wavelength of the sapphire sphere, the laser light couples to the sapphire sphere and circumnavigates the sphere by total internal reflection [24]. The elastically scattered light from the sapphire microsphere at $90^{\circ}$ is collected by a two-channel optical microscope and detected by a germanium (Ge) photodiode (D1). The elastically scattered light is separated by a beam splitter placed at an angle of $45^{\circ}$ with respect to the collected beam. The Ge photodiode signal is sent to a digital storage oscilloscope (DSO) for signal monitoring and data acquisition (DAQ). The transmitted power through the optical fiber is detected by a second Ge photodiode D2 and recorded by an optical multimeter (OMM). All the optoelectronic equipment is controlled using the general purpose interface bus (GPIB) standard. 

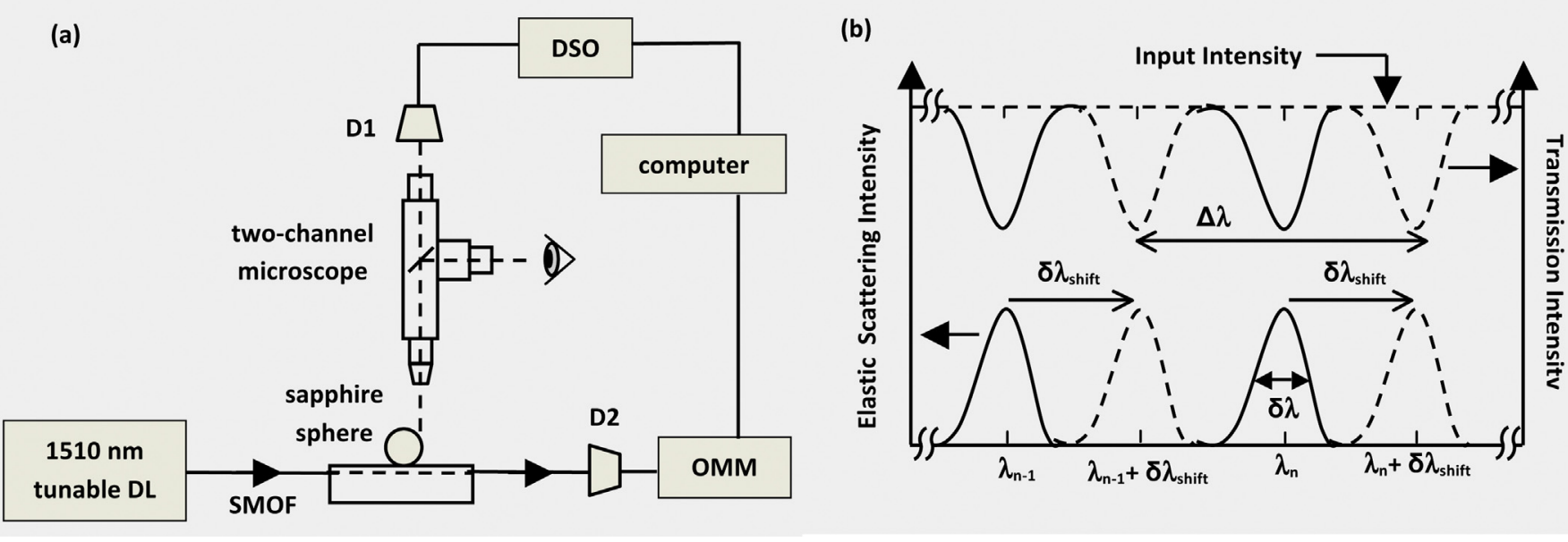

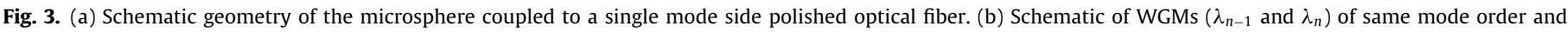
consecutive mode number, and their shift due to the sphere's surface modification.

\subsection{Detection mechanism}

The system was modeled using the Generalized Lorenz-Mie Theory (GLMT), which describes the electromagnetic scattering of an arbitrary light beam by a spherical microparticle [45]. The elastic scattering intensity from the sphere and transmittance intensity at the output of the optical fiber for transverse electric (TE) and transverse magnetic (TM) polarization were calculated for sapphire spheres before and after surface modification with biomolecules [46]. Fig. 3(b) shows the excited resonances manifested due to the circumnavigating light in the sphere. The mode spacing $\Delta \lambda$ of consecutive resonances having the same mode order (number of nodes or maxima of the intensity distribution in the radial direction) and consecutive mode number (the number of maxima between $0^{\circ}$ and $180^{\circ}$ ) in the angular intensity distribution of the mode can be calculated using the following equation: [46]

$\Delta \lambda=\frac{\lambda^{2} \arctan \sqrt{m^{2}-1}}{2 \pi a \sqrt{m^{2}-1}}$

where $m$ is the relative refractive index of the sphere (refractive index of the sphere/refractive index of the surrounding medium), $\lambda$ the wavelength of the laser source in vacuum, and $a$ is the sphere's radius. If molecules are tethered onto the sphere's surface, these molecules will induce a change of the optical properties of the sphere, especially its effective size and refractive index. As a result, the resonance wavelength will change further and result in the phenomenon of resonant shift [24]. The wavelength shift $\delta \lambda_{\text {shift }}$ can be calculated by the equation:

$\frac{\delta \lambda_{\text {shift }}}{\lambda}=\frac{\delta a}{a}$

Here, $\delta a$ is the change in sphere radius due to the interaction between the adsorbed molecules [24].

\section{Experimental results}

Fig. 4 shows the measured spectrum of the elastically scattered light from the sapphire microsphere. The $\Delta \lambda$ of the WGMs is observed to be $0.43 \mathrm{~nm}$, which is comparable with the $\Delta \lambda$ estimated using Eq. (1) for a $1 \mathrm{~mm}$ diameter sapphire microsphere with refractive index of 1.77 . For each maximum in the elastic scattering spectrum, there is a corresponding minimum in the transmittance spectrum. The minima in the transmittance spectrum correspond to a fraction of the light coupled from the fiber into the sphere.

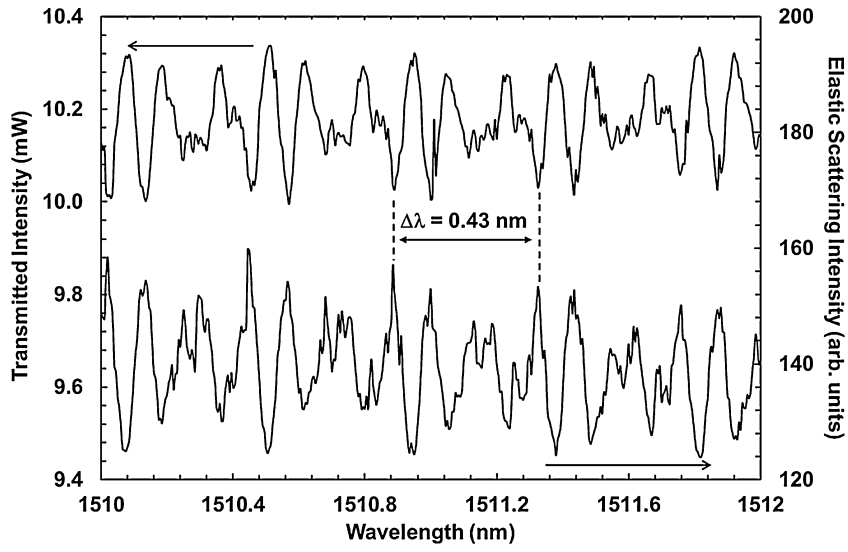

Fig. 4. Measured elastic scattering intensity (lower curve) and the corresponding transmitted intensity (upper curve) spectra from sapphire sphere of radius $500 \mu \mathrm{m}$ and refractive index 1.77 .

The linewidth $(\delta \lambda)$ of the resonances is about $0.01 \mathrm{~nm}$, which corresponds to a quality factor $Q$ of the order $10^{5}$ (using $Q=\lambda / \delta \lambda$ ).

When adding layers of PDA to the surface of the sphere, we obtain the results in Fig. 5 ( $a$ and b). Fig. 5a represents the transmitted spectra and Fig. 5b represents the elastic scattering intensity spectra for the non-modified and the modified sapphire sphere in the wavelength range from 1510 to $1512 \mathrm{~nm}$. Spectrum A represents the resonances from the non-modified sapphire sphere. Then, the sapphire sphere was polymer-coated for $2 \mathrm{~h}$ and afterwards spectrum B was collected. As a result, the resonances were red-shifted by $0.05 \mathrm{~nm}$. The spectra were collected again for $5 \mathrm{~h}$, $10 \mathrm{~h}$, and $20 \mathrm{~h}$ polymerization time, which corresponed to a shift of $0.075 \mathrm{~nm}$ (spectrum C), $0.11 \mathrm{~nm}$ (spectrum D), and $0.155 \mathrm{~nm}$ (spectrum E), respectively with a resolution of $0.005 \mathrm{~nm}$ (cfr. Table 1).

Table 1

Resonance wavelength shift and the corresponding resulting polymer film thickness as a function to polymerization time.

\begin{tabular}{llll}
\hline $\begin{array}{l}\text { Polymerization } \\
\text { time }(\mathrm{h})\end{array}$ & $\begin{array}{l}\text { Resonance } \\
\text { wavelength } \\
\text { shift }(\mathrm{nm})\end{array}$ & $\begin{array}{l}\text { Polymer film } \\
\text { thickness }(\mathrm{nm})\end{array}$ & $\begin{array}{l}\text { Quality factor } \\
(\mathrm{Q})\end{array}$ \\
\hline 0 & NA & NA & $7.5 \times 10^{4}$ \\
2 & $0.05 \pm 0.005$ & $16.6 \pm 1.7$ & $6.04 \times 10^{4}$ \\
5 & $0.075 \pm 0.005$ & $24.8 \pm 1.7$ & $5.03 \times 10^{4}$ \\
10 & $0.11 \pm 0.005$ & $36.4 \pm 1.7$ & $3.35 \times 10^{4}$ \\
20 & $0.155 \pm 0.005$ & $51.2 \pm 1.7$ & $4.3 \times 10^{4}$ \\
\hline
\end{tabular}



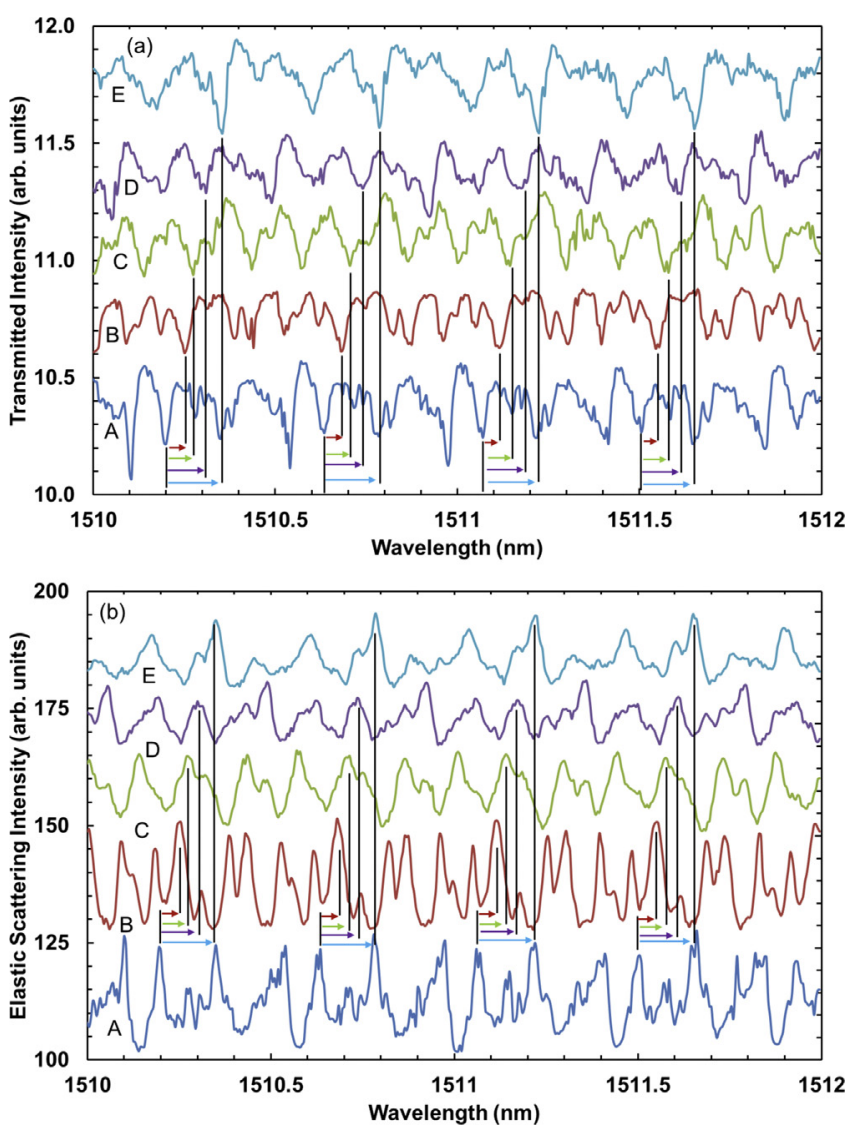

Fig. 5. Transmitted intensity (a) and elastic scattering intensity spectra (b) from a sapphire sphere with different polymerization time and the corresponding shift. A: Non-modified sphere; B: 2 h; C: 5 h; D: 10 h; and E: 20 h of polymerization.

By treating the refractive indices of PDA (1.6) and sapphire (1.77) as being almost identical and using Eq. (2), these wavelength shifts, given with a resolution of $0.005 \mathrm{~nm}$, correspond to a thickness of $16.6 \mathrm{~nm}, 24.8 \mathrm{~nm}, 36.4 \mathrm{~nm}$, and $51 \mathrm{~nm}$ (see Table 1). This correlates rather well with the thickness data determined by Lee and coworkers which are $\approx 8 \mathrm{~nm}, 25 \mathrm{~nm}, 40 \mathrm{~nm}$, and $51 \mathrm{~nm}$ for $2 \mathrm{~h}, 5 \mathrm{~h}, 10 \mathrm{~h}$ and $20 \mathrm{~h}$ polymerization time, respectively [32]. In the case of $2 \mathrm{~h}$ polymerization time, our shift $(16.6 \mathrm{~nm})$ is more pronounced than reported by Lee et al. $(8 \mathrm{~nm})$ in ref. [32]. The higher growth rate in the initial polymerization phase might be attributed to a faster nucleation due to the spherical geometry.

The spectrum of the non-modified sphere showed resonances with a quality factor of $1.5 \times 10^{5}$ while the spectra of the modified sphere showed resonances with a maximum quality factor of $6.04 \times 10^{4}$. This is possibly due to the fact that higher polymer concentrations lead to the formation of an inhomogeneous coating, with the presence of clusters [13]. This scatters the photons out of the WGM and decreases the $Q$ as shown in Table 1 [47].

Fig. 5(a) and (b) were zoomed into one resonance to study the relationship between the PDA layer thickness and the resonance quality factor. It can be seen from Fig. 6(a) and (b) that as the PDA layer thickness increases, the resonance linewidth increases.

As shown in Table 1 the quality factors of the analyzed resonances were on the same order $\left(Q \approx 10^{4}\right)$ and this verifies that the PDA layers are widely homogeneous. As the polymer thickness is increasing, the quality factor of the resonances is decreasing; this might be due to the fact that the PDA surface is becoming more heterogeneous with increasing polymer thickness. Fig. 7 represents the resulting shift in resonance wavelengths as a function of the polymer film growth time. The polymer thicknesses corresponding to
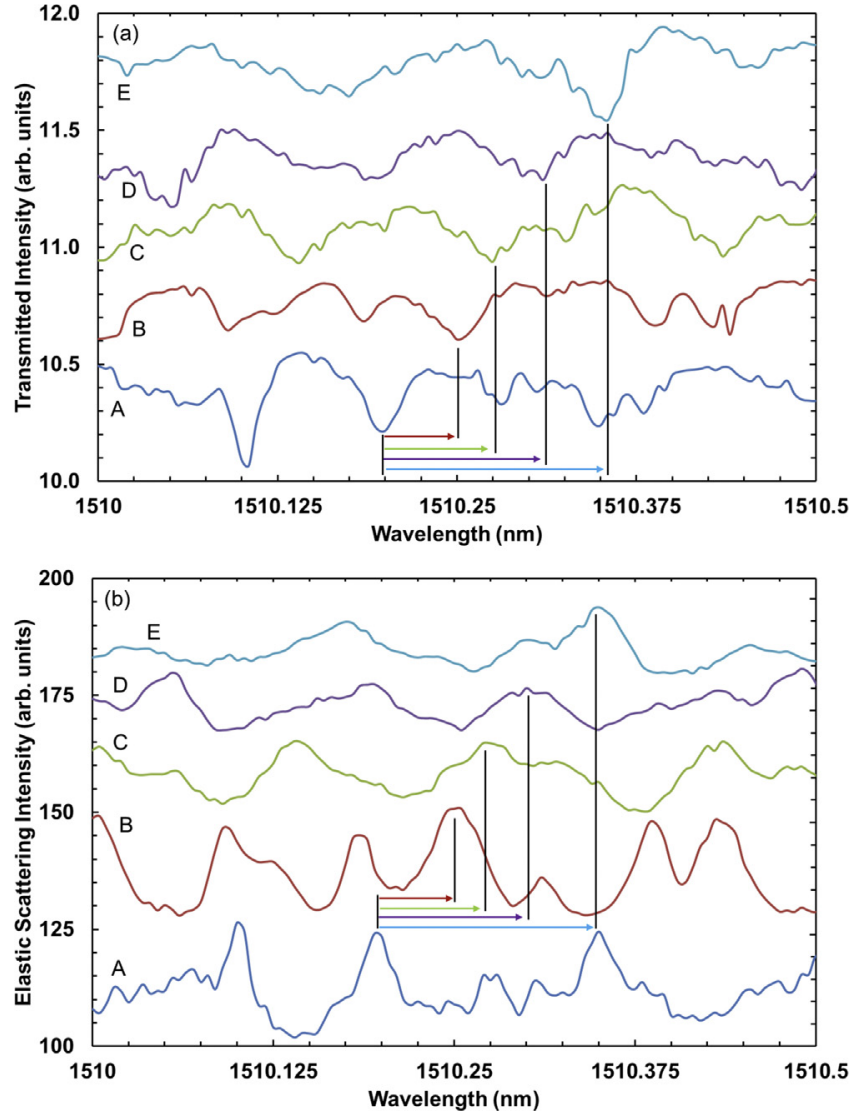

Fig. 6. Zoom of Fig. 5. Single resonance of the transmitted intensity (a) and elastic scattering intensity spectra (b) from a sapphire sphere with different polymerization times and the corresponding wavelength shift. A: Non-modified sphere; B: 2 h; C: $5 \mathrm{~h}$; D: $10 \mathrm{~h}$; and E: $20 \mathrm{~h}$ of polymerization.

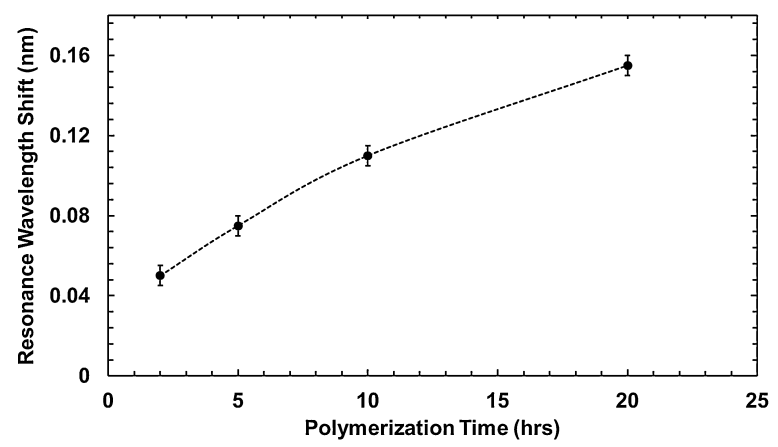

Fig. 7. Resonance wavelength shift corresponding to the sapphire surface polymerization time with PDA. Line is a guide for the eye.

the resonance wavelength shifts were calculated using Eq. (2) as shown in Table 1. As the polymerization time increases, the resonance wavelength shifts to a value, which corresponds to a thicker PDA layer on the sapphire surface.

\section{Conclusion}

We have studied the transmitted intensity and the elastic scattering intensity of a sapphire sphere (radius $500 \mu \mathrm{m}$ and 1.77 refractive index) in the wavelength range of $1510-1512 \mathrm{~nm}$. We have demonstrated the highest quality factor, in the order of $10^{5}$, up to date in a spherical resonator fabricated from single crystalline sapphire. The shift in resonance wavelength arising from coating the sphere with different thicknesses of PDA layers was studied. 
The quality factor of the resonances was studied as a function of the change in the PDA polymer thickness, and we found that it is almost preserved despite a small decrease in the coefficients. We also verified that PDA layers are homogeneous which resulted in high $Q$ factors, comparable with the values achieved with silica spheres coated with polylactic-acid (PLLA, a crystalline biodegradable polymer) [13], showing that PDA coating is a valid alternative method to silanization for coupling bioreceptors such as DNA, proteins and even cells to the sphere. The experimental feasibility of the photonic biosensing concept with sapphire microspheres was recently proven within DNA-characterization studies using a silane crosslinker between DNA and the sapphire spheres [48]. The PDA coatings described in this article are expected to offer even more experimental flexibility due to the numerous available coupling protocols between organic- and biological compounds.

\section{Authors contributions}

The experimental setup was built by M.S.M. in cooperation with D.M. All experimental measurements were performed by M.S.M. in cooperation with D.M. W.-S.Y. modified the sapphire sphere with the PDA. Biological assistance and guidance was provided by L.M. XPS measurements were performed by X.L. in cooperation with M.F. M.S.M. interpreted the optical data in close cooperation with P.W., A.S., and P.B. Insights in technical improvements of the setup were provided by P.W., A.S., and K.H. All authors have given approval to the final version of the manuscript.

\section{Acknowledgments}

This work is supported by the Life-Science Initiative Limburg and the Research Foundation Flanders FWO (Projects G.0829.09, Synthetic diamond films as platform materials for novel DNA sensors based on electronic detection techniques and G.0997.11N, Diamond-based impedimetric and nanophotonic biosensors for the detection of proteins).

\section{References}

[1] T. Strick, J.F. Allemand, V. Croquette, D. Bensimon, Twisting and stretching single DNA molecules, Prog. Biophys. Mol. Biol. 74 (2000) 115-140.

[2] U. Bockelmann, P. Thomen, B. Essevaz-Roulet, V. Viasnoff, F. Heslot, Unzipping DNA with optical tweezers: high sequence sensitivity and force flips, Biophys. J. 82 (2002) 1537-1553.

[3] J.D. Besant, J. Das, E.H. Sargent, S.O. Kelley, Proximal bacterial lysis and detection in nanoliter wells using electrochemistry, ACS Nano 7 (2013) 8183-8189.

[4] B. Lam, R.D. Holmes, J. Das, M. Poudineh, A. Sage, E.H. Sargent, S.O. Kelley, Optimized templates for bottom-up growth of high-performance integrated biomolecular detectors, Lab Chip 13 (2013) 2569-2575.

[5] S. Ingebrandt, Y. Han, F. Nakamura, A. Poghossian, M.J. Schöning, A. Offenhäusser, Label-free detection of single nucleotide polymorphisms utilizing the differential transfer function of field-effect transistors, Biosens. Bioelectron. 22 (2007) 2834-2840.

[6] A. Poghossian, M.H. Abouzar, F. Amberger, D. Mayer, Y. Han, S. Ingebrandt, A. Offenhäusser, M.J. Schöning, Field-effect sensors with charged macromolecules: characterisation by capacitance-voltage, constant-capacitance, impedance spectroscopy and atomic-force microscopy methods, Biosens. Bioelectron. 22 (2007) 2100-2107.

[7] B. van Grinsven, N. Vanden Bon, L. Grieten, M. Murib, S.D. Janssens, K. Haenen, E. Schneider, S. Ingebrandt, M.J. Schöning, V. Vermeeren, M. Ameloot, L. Michiels, R. Thoelen, W. De Ceuninck, P. Wagner, Rapid assessment of the stability of DNA duplexes by impedimetric real-time monitoring of chemically induced denaturation, Lab Chip 11 (2011) 1656-1663.

[8] B. van Grinsven, N. Vanden Bon, H. Strauven, L. Grieten, M. Murib, K.L.J. Monroy, S.D. Janssens, K. Haenen, M.J. Schöning, V. Vermeeren, M. Ameloot, L. Michiels, R. Thoelen, W. De Ceuninck, P. Wagner, Heat-transfer resistance at solid-liquid interfaces: a tool for the detection of single-nucleotide polymorphisms in DNA, ACS Nano 6 (2012) 2712-2721.

[9] Z.H. Zhong, D.L. Wang, Y. Cui, M.W. Bockrath, C.M. Lieber, Nanowire crossbar arrays as address decoders for integrated nanosystems, Science 302 (2003) 1377-1379.

[10] J.M. Nam, C.S. Thaxton, C.A. Mirkin, Nanoparticle-based bio-bar codes for the ultrasensitive detection of proteins, Science 301 (2003) 1884-1886.
[11] W.S. Yeo, D.H. Min, R.W. Hsieh, G.L. Greene, M. Mrksich, Label-free detection of protein-protein interactions on biochips, Angew. Chem. Int. Ed. 44 (2005) 5480-5483.

[12] T.P. Burg, M. Godin, S.M. Knudsen, W. Shen, G. Carlson, J.S. Foster, K. Babcock, S.R. Manalis, Weighing of biomolecules, single cells and single nanoparticles in fluid, Nature 446 (2007) 1066-1069.

[13] S. Soria, S. Berneschi, M. Brenci, F. Cosi, G.N. Conti, S. Pelli, G.C. Righini, Optical microspherical resonators for biomedical sensing, Sensors 11 (2011) 785-805.

[14] J.L. Nadeau, V.S. Iltchenko, D. Kossakovski, G.H. Bearman, L. Maleki, High-Q whispering-gallery mode sensor in liquids, in: Conference on Laser Resonators and Beam Control V, San Jose, California, 2002, pp. 172-180.

[15] J.Y. Lin, A.D. Stuparu, M.D. Huntington, M. Mrksich, T.W. Odom, Nanopatterned substrates increase surface sensitivity for real-time biosensing, J. Phys. Chem. C 117 (2013) 5286-5292.

[16] J.D. Suter, I.M. White, H.Y. Zhu, H.D. Shi, C.W. Caldwell, X.D. Fan, Label-free quantitative DNA detection using the liquid core optical ring resonator, Biosens. Bioelectron. 23 (2008) 1003-1009.

[17] Z.B. Bahși, A. Büyükaksoy, S.M. Ölmezcan, F. Simșek, M.H. Aslan, A.Y. Oral, A novel label-free optical biosensor using synthetic oligonucleotides from $E$. coli 0157:H7: elementary sensitivity tests, Sensors 9 (2009) 4890-4900.

[18] M. Baaske, F. Vollmer, Optical resonator biosensors: molecular diagnostic and nanoparticle detection on an integrated platform, ChemPhysChem 13 (2012) 427-436.

[19] R. Chen, V.D. Ta, H. Sun, Bending-induced bidirectional tuning of whispering gallery mode lasing from flexible polymer fibers, ACS Photonics 1 (2014)11-16.

[20] A. Serpengüzel, S. Arnold, G. Griffel, Excitation of resonances of microspheres on an optical fiber, Optics Lett. 20 (1995) 654-656.

[21] K. De Vos, I. Bartolozzi, E. Schacht, P. Bienstman, R. Baets, Silicon-on-insulator microring resonator for sensitive and label-free biosensing, Opt. Express 15 (2007) 7610-7615.

[22] R. Kirchner, M.K. Kaiser, B. Adolphi, R. Landgraf, W.J. Fischer, Chemical functional polymers for direct UV assisted nanoimprinting of polymeric photonic microring resonators, Phys. Status Solidi A 208 (2011) 1308-1314.

[23] K. De Vos, J. Girones, S. Popelka, E. Schacht, R. Baets, P. Bienstman, SOI optical microring resonator with poly(ethylene glycol) polymer brush for label-free biosensor applications, Biosens. Bioelectron. 24 (2009) 2528-2533.

[24] F. Vollmer, S. Arnold, Whispering-gallery-mode biosensing: label-free detection down to single molecules, Nat. Methods 5 (2008) 591-596.

[25] V.S. Ilchenko, A.B. Matsko, Optical resonators with whispering-gallery modes - part II: applications, IEEE J. Sel. Top. Quantum Electron. 12 (2006) 15-32.

[26] F. Vollmer, D. Braun, A. Libchaber, M. Khoshsima, I. Teraoka, S. Arnold, Protein detection by optical shift of a resonant microcavity, Appl. Phys. Lett. 80 (2002) 4057-4059.

[27] V. Vermeeren, S. Wenmackers, M. Daenen, K. Haenen, O.A. Williams, M. Ameloot, M. Vandeven, P. Wagner, L. Michiels, Topographical and functional characterization of the ssDNA probe layer generated through EDCmediated covalent attachment to nanocrystalline diamond using fluorescence microscopy, Langmuir 24 (2008) 9125-9134.

[28] T. Strother, W. Cai, X.S. Zhao, R.J. Hamers, L.M. Smith, Synthesis and characterization of DNA-modified silicon (111) surfaces, J. Am. Chem. Soc. 122 (2000) 1205-1209.

[29] B.J.M. Hausmann, B. Shields, Q.M. Quan, P. Maletinsky, M. McCutcheon, J.T Choy, T.M. Babinec, A. Kubanek, A. Yacoby, M.D. Lukin, M. Lončar, Integrated diamond networks for quantum nanophotonics, Nano Lett. 12 (2012) 1578-1582.

[30] V.S. Ilchenko, A.M. Bennett, P. Santini, A.A. Savchenkov, A.B. Matsko, L. Maleki, Whispering gallery mode diamond resonator, Opt. Lett. 38 (2013) 4320-4323.

[31] H. Kawarada, A.R. Ruslinda, Diamond electrolyte solution gate FETs for DNA and protein sensors using DNA/RNA aptamers, Phys. Status Solidi A 208 (2011) 2005-2016.

[32] H. Lee, S.M. Dellatore, W.M. Miller, P.B. Messersmith, Mussel-inspired surface chemistry for multifunctional coatings, Science 318 (2007) 426-430.

[33] S.M. Kang, N.S. Hwang, J. Yeom, S.Y. Park, P.B. Messersmith, I.S. Choi, R. Langer, D.G. Anderson, H. Lee, One-step multipurpose surface functionalization by adhesive catecholamine, Adv. Funct. Mater. 22 (2012) 2949-2955.

[34] M.E. Lynge, R. van der Westen, A. Postma, B. Stadler, Polydopamine - a nature-inspired polymer coating for biomedical science, Nanoscale 3 (2011) 4916-4928.

[35] O. Pop-Georgievski, N. Neykova, V. Proks, J. Houdkova, E. Ukraintsev, J. Zemek, A. Kromka, F. Rypacek, Polydopamine-modified nanocrystalline diamond thin films as a platform for bio-sensing applications, Thin Solid Films 543 (2013) $180-186$.

[36] Y. Wan, D. Zhang, Y. Wang, P. Qi, B.R. Hou, Direct immobilisation of antibodies on a bioinspired architecture as a sensing platform, Biosens. Bioelectron. 26 (2011) 2595-2600.

[37] K. Kang, I.S. Choi, Y. Nam, A biofunctionalization scheme for neural interfaces using polydopamine polymer, Biomaterials 32 (2011) 6374-6380.

[38] H. Lee, J. Rho, P.B. Messersmith, Facile conjugation of biomolecules onto surfaces via mussel adhesive protein inspired coatings, Adv. Mater. 21 (2009) 431-434.

[39] K. Sun, Y.Y. Xie, D.K. Ye, Y.Y. Zhao, Y. Cui, F. Long, W. Zhang, X.Y. Jiang Mussel-inspired anchoring for patterning cells using polydopamine, Langmuir 28 (2012) 2131-2136.

[40] J.R. Schwesyg, T. Beckmann, A.S. Zimmermann, K. Buse, D. Haertle, Fabrication and characterization of whispering-gallery-mode resonators made of polymers, Opt. Express 17 (2009) 2573-2578. 
[41] A.G. Mamalis, J.J. Ramsden, A.I. Grabchenko, L.A. Lytvynov, V.A. Filipenko, S.N. Lavrynenko, A novel concept for the manufacture of individual sapphiremetallic hip joint endoprostheses, Biol. Phys. Chem. 6 (2006) 113-117.

[42] T. Takahashi, T. Sato, R. Hisanaga, O. Miho, Y. Suzuki, M. Tsunoda, K.I. Nakagawa, Long-term observation of porous sapphire dental implants, Bull. Tokyo Dent. Coll. 49 (2008) 23-27.

[43] M.R. Rieger, W.K. Adams, G.L. Kinzel, M.O. Brose, Alternative materials for three endosseous implants, J. Prosthet. Dent. 61 (1989) 717-722.

[44] G.Q. Zhou, Y.J. Dong, J. Xu, H.J. Li, J.L. Si, X.B. Qian, X.Q. Li, $\varphi 140$ mm Sapphire crystal growth by temperature gradient techniques and its color centers, Mater. Lett. 60 (2006) 901-904.

[45] G. Gouesbet, J.A. Lock, Rigorous justification of the localized approximation to the beam-shape coefficients in generalized Lorenz-Mie theory. II. Off-axis beams, J. Opt. Soc. Am. A 11 (1994) 2516-2525.

[46] M.S. Murib, A.Q. Tran, W. De Ceuninck, M.J. Schöning, M. Nesládek, A. Serpengüzel, P. Wagner, Analysis of an optical biosensor based on elastic light scattering from diamond-, glass-, and sapphire microspheres, Phys. Status Solidi A 209 (2012) 1804-1810.

[47] D.W. Vernooy, V.S. Ilchenko, H. Mabuchi, E.W. Streed, H.J. Kimble, High-Q measurements of fused-silica microspheres in the near infrared, Opt. Lett. 23 (1998) 247-249.

[48] M.S. Murib, W.-S. Yeap, D. Martens, P. Bienstman, W. De Ceuninck, B. van Grinsven, M.J. Schöning, L. Michiels, K. Haenen, M. Ameloot, A. Serpengüzel, P. Wagner, Photonic detection and characterization of DNA using sapphire microspheres, J. Biomed. Opt. 19 (2014) (art. no. 097006).

\section{Biographies}

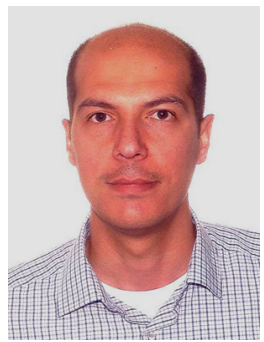

M.S. Murib received his B.Sc in Physics from Lebanese University, Faculty of Science, Hadath, Lebanon, in 2005. He joined the MSc program in Physics of Koç University in 2007, as a teaching/research assistant during which he worked with Prof. Ali Serpengüzel at the Koç University Microphotonics Research Laboratory. In 2010, he joined the Bios group at the Institute for Materials Research (IMO) of Hasselt University, Belgium for his PhD during which he worked with Prof. Patrick Wagner on bioelectronics and biophotonic sensors. He received his Ph.D. in February 2014.

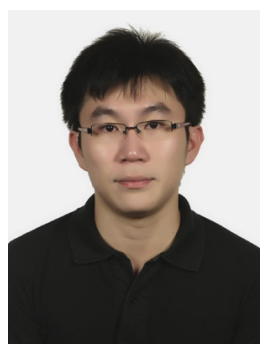

W.S. Yeap obtained his M.Sc. degree in 2009 at National University of Singapore and is presently a Ph.D. student in Prof. Ken Haenen's group. His research interests include surface functionalization of diamond, diamond electrochemistry, and (bio)chemical sensor. He is the author and co-author of several research articles in these areas.

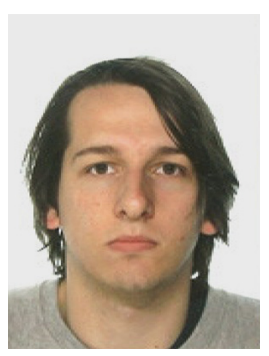

D. Martens received the master's degree in engineering physics from Ghent University in 2012. Since 2012, he is working toward the Ph.D. degree with the Photonic Research Group, Ghent University where he is involved in the Pocket project. His current research interests include integrated photonic biosensors, the VNIR wavelength range, silicon nitride and on-chip spectral filters.

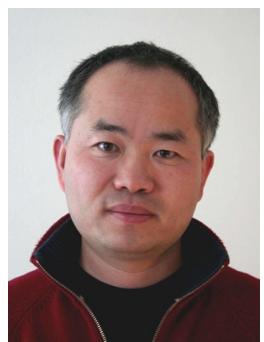

X. Liu obtained his Bachelor degree in Physics from Lanzhou University, China in 1993, and the Ph.D. in Physics from Leibniz IFW-Dresden/TU-Dresden in October 2003. After that, he spent several years in Austria and the US working on different spectroscopies. In 2011, he joined the surface physics and chemistry division at Department of Physics, Chemistry, and Biology (IFM), Linköping University, Sweden. His main research topic focuses on the electronic and optical properties of nanomaterials, organic molecule/polymer and their interface properties.

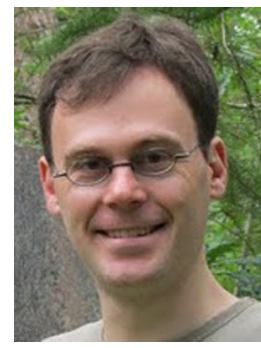

tion processing.

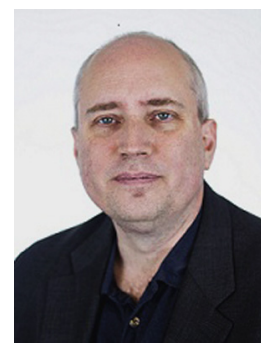

Mats Fahlman was born 1967 in Uppsala, Sweden, and received his M.S. in Electrical Engineering and his Ph.D. in Surface Physics and Chemistry at Linköping University in 1991 and 1995, respectively. He became Associate Professor and later Professor in experimental physics at Linköping University in 2000 and 2005, respectively, and is the Professor of Surface Physics and Chemistry since August 2008. He is currently active in the fields of organic electronic and organic spintronics with a focus on interface effects

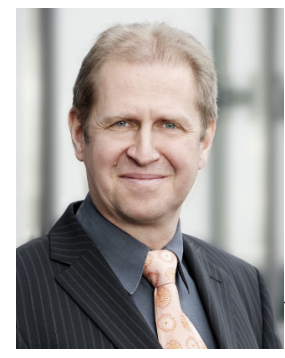

M.J. Schöning received his diploma degree in electrical engineering (1989) and his Ph.D. in the field of semiconductor-based microsensors for the detection of ions in liquids (1993), both from the Karlsruhe University of Technology. In 1989, he joined the Institute of Radiochemistry at the Research Centre Karlsruhe. Since 1993 he has been with the Institute of Thin Films and Interfaces (now, Institute of Bio- and Nanosystems) at the Research Centre Jülich, and since 1999 he was appointed as full professor at Aachen University of Applied Sciences, Campus Jülich. Since 2006, he serves as a director of the Institute of Nano- and Biotechnologies (INB) at the Aachen University of Applied Sciences. His main research subjects concern silicon-based chemical and biological sensors, thin-film technologies, solid-state physics, microsystem and nano(bio-)technology.

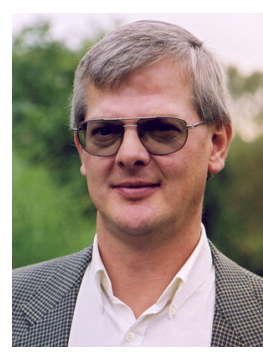

L. Michiels has a Ph.D. in biochemistry and is full professor of the faculty of Medicine and Life Sciences at Hasselt University. He is responsible for the Bionanotechnology research program in the Biomedical research Institute BIOMED and his research is focusing on the integration of disease biomarkers in innovative electronic biosensing platforms for the development of new biomedical applications.

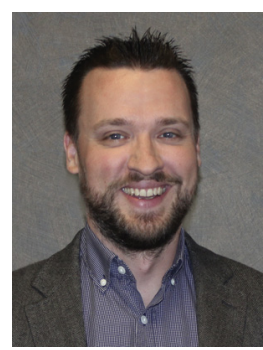

K. Haenen obtained a M.S. in Physics from KU Leuven in 1997 and a Ph.D. in Physics from Hasselt University in 2002. In 2004, after a postdoc period at NIMS, Japan, he became the group leader of Wide Band Gap Materials at the Institute for Materials Research (IMO) from Hasselt University. This was followed by his appointment as professor of experimental physics in 2008. Haenen's research focusses on different fundamental and applied aspects of CVD diamond, including combinations with other materials like AlN, BN, and small molecules. He currently serves as one of the Meeting Chairs for the 2015 MRS Spring Meeting, and is also involved in several conferences on diamond and carbon materials (DCM, NDNC, SBDD) as Chair or Organiser. Ken Haenen is Editor of Diamond and Related Materials and annual Guest Editor of Physica Status Solidi A for Special Issues on diamond and nanocarbons. 


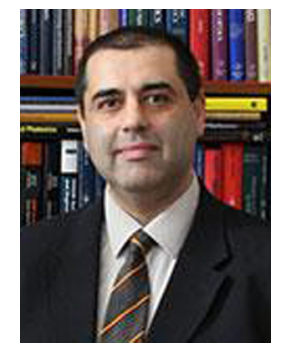

A. Serpengüzel received his Ph.D. from Yale University in applied physics in 1992. At Yale University Center for Laser Diagnostics, he worked on nonlinear optical light scattering from microdroplets. Later on, he joined Polytechnic University, Microparticle Photophysics Laboratory as a postdoctoral research associate, where he performed the first coupling experiment of solid microspheres to opti-

cal fibers. Afterwards, he joined Bilkent University as an assistant professor of physics, where he concentrated his research on the optoelectronic properties of semiconductor microcavities and semiconductor microdevices. He is currently a professor of physics and the director of the Microphotonics Research Laboratory, he established in 2000 at Koç University. His current research focuses on silicon photonics, optical microcavities, and waveguides toward applications in optical fiber communications. Other research interests include optical spectroscopy in complex media, nanophotonics, linear and nonlinear optics, and laser diagnostics toward applications in remote sensing and combustion. He is an SPIE fellow, IEEE and OSA senior member and Sigma-Xi member.

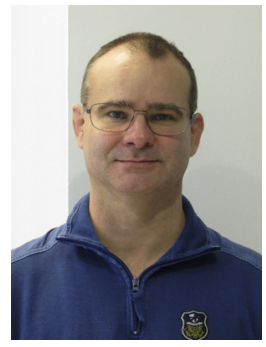

P. Wagner obtained his Ph.D. in physics in 1994 at the Technical University Darmstadt for his studies on superconductors. Between 1995 and 2001 he was a postdoctoral researcher at the Catholic University Leuven focussing on magnetic oxides. In 2001, he founded the BIOSensor group within the Institute for Materials Research at Hasselt University. His research team develops label-free sensing techniques for the detection and characterization of small molecules, proteins, nucleic acids, and circulating cells. In 2014 he moved back to KU Leuven as a full professor for bio- and soft-matter physics with a special focus on bio-functional surfaces. P. Wagner received several scientific distinctions including a Marie-Curie fellowship of the European Union (1996 - 1998) and a Methusalem grant of the Flemish Government $(2008-2014)$ 